\title{
CONSTRUÇÃO DE AMBIENTES EDUCACIONAIS COM REALIDADE AUMENTADA: PROCESSO CENTRADO NO USUÁRIO
}

\author{
Paula Teixeira Nakamoto, Instituto Federal do Triângulo Mineiro, \\ paula@iftriangulo.edu.br
}

Gilberto Arantes Carrijo, Universidade Federal de Uberlândia, gilberto@ufu.br

Alexandre Cardoso, Universidade Federal de Uberlândia, alexandre@ufu.br

\begin{abstract}
Resumo: Nos últimos anos, uma proliferação de novas tecnologias vem surgindo para melhorar/facilitar o ensino e a assimilação das informações. Uma dessas tecnologias é a Realidade Aumentada - RA que enriquece o ambiente físico (real) com objetos virtuais. No entanto, é notavelmente maior a complexidade em projetá-los e usá-los do que interfaces/softwares $2 D$, onde o usuário, na maioria das vezes, já está habituado com as tecnologias. Projetistas de ambientes educacionais com RA precisam de diretrizes para tornar esses sistemas mais usáveis e diminuir assim a carga cognitiva, pois as orientações convencionais para construção de softwares, somente estão disponíveis para ambientes 2D. Dessa forma, este trabalho tem o objetivo de apresentar uma investigação que resultou em uma metodologia de Análise de Requisitos, específica para construção de objetos educacionais com RA, que visa estudar o modo como o usuário se comporta com as novas tecnologias usadas em RA (câmeras, capacetes, marcadores) e a junção do real com o virtual, construindo uma metodologia totalmente centrada no usuário.
\end{abstract}

Palavras-chave: Objetos de Aprendizagem, Interação Humano-Computador, Engenharia de Usabilidade, Realidade Aumentada, Análise de Requisitos.

Abstract: In the last years, a proliferation of new technologies has appearing to improvelfacilitate the instruction and the assimilation of the information. One of those technologies is the Augmented Reality AR that enriches the physical (real) environment with virtual objects. However, it is substantially larger the complexity to project and use them than 2D interfaces/softwares, where the user, most of the time, already habituated with the technologies. The AR environment educations designers needs guidelines to convert these system more usable and thus reduce the cognitive weight, because the conventional orientations to software development are only available for $2 D$ environment. AR engineers needs guidelines to aid them in the development of those emerging systems, because the conventional orientations are only available for the $2 D$ environments. In that way, this work has the objective to present an investigation that resulted in an methodology of Requirements Analysis, specific to the development of AR education objects, tthat seeks to study the way as the user behaves with the new technologies used in AR (cameras, helmets, markers) and the join of the real with the virtual, building an usable methodology and totally centered in the user.

Key-words: Environment Educations, Human-Computer Interaction, Usability Engineering, Augmented Reality, Requirements Analysis.

V. $7 \mathrm{~N}^{\circ} 3$, dezembro, 2009 


\section{INTRODUÇÃ̃O}

Nos últimos anos, uma proliferação de novos sistemas vem surgindo para melhorar/facilitar o ensino e a assimilação das informações (Laudon \& Laudon, 2007). Pesquisas recentes, (Bastos, 2007), (Schoenfelder \& Schmalstieg, 2008) mostram que muitos desses ambientes educacionais tem sido desenvolvidos com as mais novas tecnologias do mercado. Uma dessas tecnologias emergentes que esta sendo muito utilizada para o ensino chama-se Realidade Aumentada (RA), que é a sobreposição de objetos virtuais tridimensionais, gerados por computador, em um ambiente real, por meio de algum dispositivo tecnológico. RA enriquece o ambiente físico com objetos virtuais, ampliando o entendimento do ambiente real. Esses sistemas podem ser usados tanto em plataformas sofisticadas quanto em plataformas populares e estão sendo utilizados em várias áreas, como: ensino, marketing, indústria, saúde e etc. (Swan \& Gabbard, 2005).

Apesar do aumento na utilização de ambientes de RA, eles apresentam, principalmente, no ensino e aprendizagem, um grande desafio: projetar interfaces para o usuário, pois projetistas de RA não tem estabelecido um conjunto de diretrizes ou heurísticas para ajudar na implementação. (Jeon et al., 2006), (Kulas et al., 2004), (Fernandes \& Sanches, 2008), (Vanderdonckt et al., 2004), (Bonanni et al., 2005).

Segundo Wang \& Reevers (2007) a usabilidade não tem recebido um foco adequado, não se preocupando com os diferentes usuários e suas diferentes capacidades na utilização das tecnologias. Muitos designers instrucionais criam objetos de aprendizagem sem se preocuparem se esse recurso é adequado para o público que vai utilizá-lo. Eles também, não se preocupam, em realizar uma pesquisa, uma análise de requisitos antes da construção do software, deixando muitas vezes, a avaliação, somente para o final do desenvolvimento do software, o que é muito mais complicado e oneroso.

Costabile et al. (2005) afirmam que aplicações para o ensino, tais como, objetos de aprendizagem, devem ser fáceis de serem usadas. Caso contrário, o tempo do estudante será desperdiçado com a aplicação, ao invés de ser aproveitado para aprendizagem. Costabile et al. (2005) complementam que pessoas se recusam a usar uma interface de um sistema que seja rígida, lenta e desagradável e acabam interrompendo o curso. Essa facilidade de uso está relacionada à usabilidade.

A usabilidade deve ser trabalhada desde a criação do projeto, mas desenvolvedores muitas vezes não possuem recursos suficientes, tempo ou conhecimentos adequados para identificar as necessidades de usabilidade de todos os usuários potenciais (Roger et al., 2007). Porém, essa consciência é parte importante do processo de desenvolvimento do software (Engenharia de Usabilidade) e exige mais estudo e atenção daqueles que desenvolvem os ambientes de aprendizagem.

Quando examinadas várias abordagens de engenharia de usabilidade tradicionais e especificadamente projetos e atividades de avaliação, na maioria dos casos, atividades de projeto são alavancadas por metáforas existentes, guias ou padrões. Contudo, em casos onde a tecnologia provê abordagens modernas para interação dos usuários ou fundamentalmente altera o modo dos usuários perceberem o espaço de interação, como acontece com RA, projetistas, frequentemente, pouco compreendem as ramificações perceptivas ou cognitivas do projeto, não sabendo como cada usuário irá se adaptar às condições do ambiente do sistema (como manipulação de ambientes reais e virtuais ao mesmo tempo, uso de capacetes, uso de câmeras de vídeo, marcadores e etc.). 
Como resultado, processos/metodologias são necessários para ajudar os projetistas de novas tecnologias, como RA, a criar e avaliar os projetos, ganhando um melhor entendimento dos efetivos parâmetros e determinando em que condições esses parâmetros devem ser aplicados. Sem esses processos, aplicações desenvolvidas usando a engenharia de usabilidade tradicional podem ou não melhorar a usabilidade do sistema (Amado, 2007).

Então, para facilitar o desenvolvimento desses sistemas educacionais e aumentar a sua usabilidade, esse trabalho, em andamento, apresenta a criação de uma metodologia de análise de requisitos específica para construção de objetos educacionais com RA. Logo, para que isso seja possível o projeto deve ser totalmente centrado no usuário, pois na análise do domínio um dos maiores ganhos é entender o ponto de vista do usuário. Vale salientar que este trabalho faz parte de um trabalho maior que envolve o estabelecimento de um guia que ajudará os engenheiros de usabilidade a decidir como proceder na escolha da interface e dos equipamentos utilizados no ambiente.

Este artigo está organizado da seguinte forma. Na segunda seção faz-se uma breve descrição sobre Realidade Aumentada. A terceira seção traz algumas considerações sobre a importância da Usabilidade e Engenharia de Usabilidade. A quarta seção demonstra uma proposta de metodologia de análise de requisitos para RA e a quinta seção resume o estudo de caso realizado e apresenta alguns resultados iniciais. Por fim, na sexta seção, encontram-se as conclusões.

\section{REALIDADE AUMENTADA}

A Realidade Aumentada - RA é um caso particular das realidades mistas. Estas conjugam realidade com virtualidade, seja através da introdução de objetos reais no mundo virtual (virtualidade aumentada), seja adicionando objetos virtuais à realidade, (realidade aumentada) como forma de complementar em lugar de substituir (Azuma, 1993). No entanto, na literatura geralmente encontra-se o termo realidade aumentada com o significado de realidade mista. A vantagem da RA é que ela é um sistema que aumenta a quantidade de informações disponíveis aos ambientes existentes, onde as informações carregadas pelos objetos virtuais ajudam um usuário a executar tarefas do mundo real (Swan \& Gabbard, 2005).

Os sistemas de RA podem ser classificados conforme o tipo de display utilizado. Os dois tipos mais utilizados são:

- O sistema de visão ótica direta utiliza óculos ou capacetes com lentes que permitem o recebimento direto da imagem real, ao mesmo tempo em que possibilitam a projeção de imagens virtuais devidamente ajustadas com a cena real;

- O sistema de visão por vídeo baseado em monitor utiliza uma webcam (câmera de vídeo) para capturar a cena real. Depois de capturada, a cena real é misturada com os objetos virtuais gerados por computador e apresentada no monitor. Os objetos virtuais geralmente são gerados através dos marcadores de referência, possibilitando que o programador acrescente objetos virtuais sobre estes marcadores no mundo real.

Dos dois tipos de sistemas de RA apresentados, o mais utilizado para a criação de ambientes educacionais (Zorzal et al., 2008; Luz et al., 2008) é o sistema de visão por vídeo, pois os equipamentos são mais acessíveis e mais simples de serem utilizados. Esses equipamentos, geralmente, estão disponíveis em todas as escolas e faculdades. Já

V. $7 \mathrm{~N}^{\mathrm{o}}$ 3, dezembro, 2009 


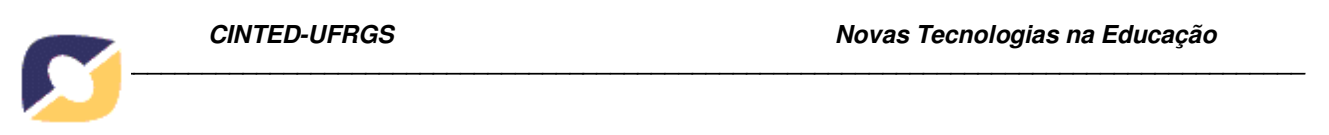

no sistema de visão ótica direta, como o usuário utiliza um capacete, nem todos se adaptam ao sistema, pois na sua utilização pode-se constatar em algumas pessoas cansaço, tontura ou perda de percepção de profundidade (Jones et al., 2008).

Considerando as informações anteriores, é extremamente importante fazer uma apurada análise de requisitos e uma pesquisa comportamental dos usuários para escolher corretamente o tipo de sistema de RA que será utilizado e para superar alguns desafios da tecnologia, que são:

- Combinar os dispositivos de entrada/saída que melhor se adaptem à tarefa;

- Facilitar o seu uso e aprendizagem;

- Permitir um elevado desempenho do utilizador e uma inerente satisfação.

\section{ENGENHARIA DE USABILIDADE}

Um aspecto indispensável para o desenvolvimento de qualquer produto ou sistema é a preocupação em torná-los o mais utilizável possível. Infelizmente, muitos dos sistemas não são pensados para os utilizadores reais, mas sim, para cumprir funções, o que do ponto de vista do funcionamento do produto está perfeitamente bom. Com o intuito de observar melhor as necessidades dos utilizadores, é necessário integrar parâmetros ou métodos que analisem minuciosamente o usuário, testem e avaliem os sistemas durante todo o seu ciclo e não somente no final do desenvolvimento, bem como, depois de implementado, assegurando, assim, a satisfação da maioria das expectativas dos utilizadores (Preece et al., 2005).

Logo, a Engenharia de Usabilidade é a utilização de princípios de engenharia de forma a obter produtos fáceis de utilizar, economicamente viáveis e que suportem trabalho real de uma forma eficaz, eficiente e promovendo a satisfação subjetiva. Usabilidade é um dos quesitos mais importantes em uma interface, uma vez que, o sucesso ou fracasso de um sistema dependerá de fatores como a facilidade de aprendizado do usuário no uso com a ferramenta, flexibilidade e robustez de sua interação.

A norma internacional ISO 13407 (ISO, 1999), Usability Engineering, define Engenharia de Usabilidade e fornece orientações para atingir a qualidade ao longo do ciclo de vida de sistemas interativos computacionais. Todavia, ela foi desenvolvida para ser aplicada em ambientes 2D, não sendo suficiente para garantir um alto nível de usabilidade em interfaces de tecnologias emergentes, como RA.

\section{METOLOGIA PROPOSTA}

A metodologia proposta nesse trabalho foi derivada de pesquisas em interação humano-computador, Engenharia de Usabilidade em RA e objetos de aprendizagem. Elas detalharam as fases do comportamento interativo e exigências de design para apoiar as fases do processo. Também foi derivada de experiências com o usuário na apresentação de novas tecnologias antes da realização da análise do domínio e de uma minuciosa análise de requisitos para avaliar o perfil do usuário que irá utilizar o objeto de aprendizagem.

O modelo, apresentado na figura 1, adota e incorpora critérios de usabilidade, enfoque no usuário e iteratividade de projeto, assim como implicações no seu processo de desenvolvimento. 


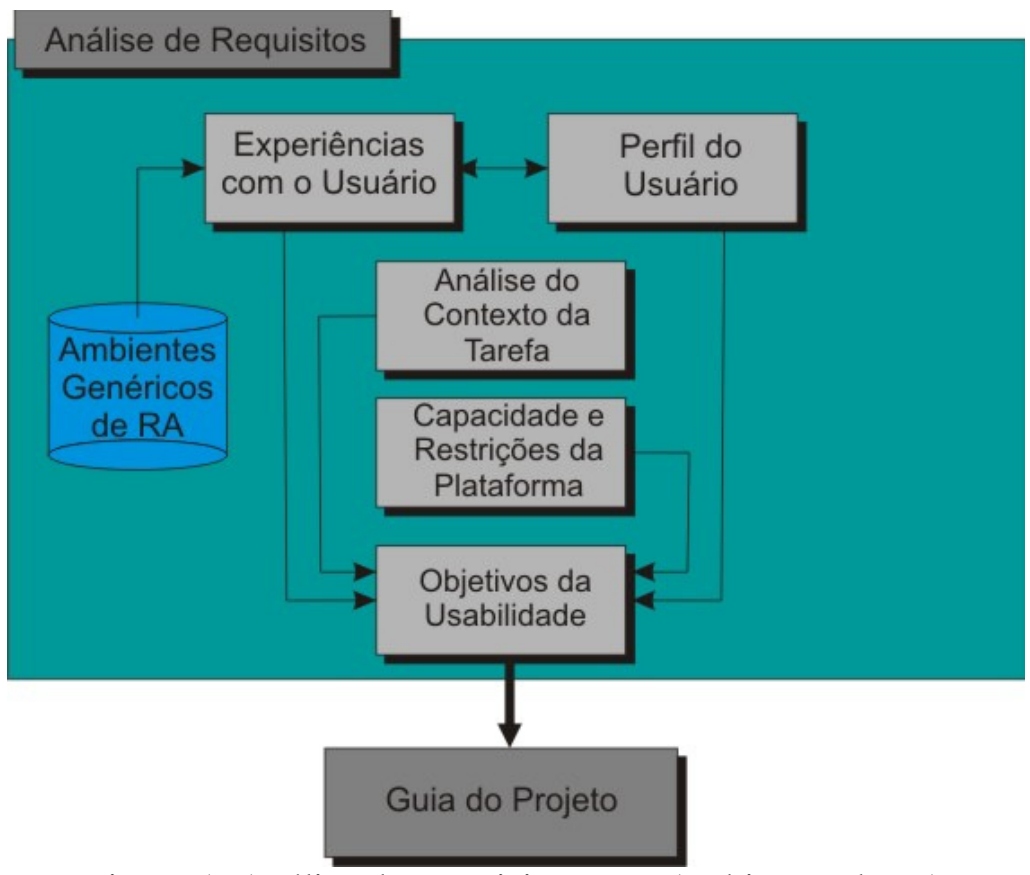

Figura 1. Análise de Requisitos para Ambientes de RA

O modelo de Análise de Requisitos para sistemas RA adotado é composto das etapas explicadas abaixo:

1. Ambientes Genéricos de RA: É um banco de dados com vários exemplos de ambientes educacionais implementados com RA. No banco de dados encontram-se ambientes com a utilização de capacetes (sistema de visão ótica direta), ambientes com a utilização de webcams (sistema de visão por vídeo) e ambientes com e sem a utilização de marcadores para a geração de imagens virtuais.

2. Experiências com Usuário: Essa etapa é realizada em paralelo com a análise do perfil do usuário. O projetista escolhe dois softwares educacionais do banco de dados de ambientes genéricos de RA. Um será do tipo visão ótica direta e o outro do tipo visão por vídeo ou dois do tipo visão por vídeo. Os softwares escolhidos são o que mais se assemelham ao sistema que será desenvolvido. Por exemplo, se for desenvolvido um software para a área da saúde, se disponível, será selecionado um ambiente da mesma área. Esse sistema é apresentado para um pequeno grupo de estudantes que possuem o mesmo perfil dos estudantes que irão utilizar o ambiente educacional que será construído. Os detalhes do estudo do perfil do usuário serão explicados no próximo item. O projetista analisa o seu comportamento com a utilização de novas tecnologias e a manipulação das informações reais junto com as informações virtuais. Após a experiência do usuário com o ambiente, ele responderá um questionário. Essas informações facilitarão o desencadear do projeto, pois o projetista, conhecendo o comportamento do usuário com a tecnologia, escolherá o tipo correto de ambiente e os equipamentos que os usuários se adaptarão melhor. Enfim, será desenvolvido um ambiente educacional que necessitará de menos 
treinamento, será mais usável e específico para o grupo de usuários que irão utilizá-lo.

3. Análise do perfil do usuário: Para cada tipo de usuário previsto, os projetistas devem conhecer seus atributos pessoais (faixa etária, sexo, limitações, motivação) e suas habilidades e competências (na tarefa, na organização, no uso de capacetes, na utilização de ambientes 3D e em sistemas informatizados).

4. Análise do contexto da tarefa: Para cada tarefa a ser apoiada pelo sistema, os projetistas devem conhecer os objetivos e resultados, a estrutura, a duração, as dependências, os custos, a carga mental, as interrupções, os incidentes etc. Por exemplo, a claridade do ambiente pode ter impactos na utilização de sistemas de RA.

5. Análise das capacidades e restrições da plataforma: Devem ser examinadas as possibilidades e restrições em termos de equipamentos (capacetes, webcam), sistemas operacionais e aplicativos disponíveis para o funcionamento do sistema. Por exemplo, se não existe capacete, na experiência com o usuário serão escolhidos dois sistemas do tipo visão por vídeo.

6. Usabilidade: Nível de usabilidade esperado para o sistema de acordo com os princípios gerais do projeto. Essa especificação é feita nos termos de valores mínimos admissíveis para os fatores básicos de usabilidade: eficácia, eficiência e satisfação do usuário, principalmente.

7. Guia do Projeto: Registra todas as decisões tomadas nas atividades da Análise de Requisitos e servirá como guia para a construção do projeto, construção do protótipo, implementação do sistema e futuras avaliações do ambiente.

\section{CONCLUSÕES}

Uma das principais contribuições deste trabalho está no fato de apresentar uma metodologia de análise de requisitos específica para construção de ambientes educacionais com RA. Vale ressaltar que uma completa e específica análise de requisitos impacta positivamente na elaboração de qualquer sistema, sendo, portanto, um mecanismo essencial de apoio à elaboração de objetos educacionais. Do mesmo modo, essas orientações poderão contribuir para o processo de ensino e aprendizagem, pois tornarão os objetos de aprendizagem mais fáceis de serem usado. De modo geral, a falta e limitações de guias nortearam a necessidade de pesquisas na área e a criação de uma metodologia específica, visto a grande utilização de sistemas de RA. Portanto, concentrar atenção na análise do domínio e no comportamento do usuário, claramente contribui para a qualidade dos sistemas de software. Vale salientar que a metodologia estabelecida neste trabalho ainda esta em desenvolvimento e que além dela, pretende-se criar um guia completo para auxiliar os projetistas no desenvolvimento de softwares educacionais com RA. Essa metodologia foi proposta seguindo a idéia central da norma padrão internacional ISO 13407 (ISO, 1999), que tem como princípio, focalizar desde o início os usuários e as tarefas que desenvolvem num determinado ambiente. 


\section{REFERÊNCIAS BIBLIOGRÁFICAS}

\section{Livro}

LAUDON, K ; LAUDON, J. Information Systems. Prentice Hall, 2007

PREECE, J.; ROGERS, Y.; SHARP, H. Design de Interação - Além da Interação Homem - Computador. Artmed, 2005.

WANG, C.; REEVES, T. The Meaning of Culture in Online Education: Implications for Teaching, Learning, and Design. In Andrea Edmundson, Globalized E-Learning Cultural Challenges. Information Science Publishing, Hershey, PA, USA. p 1-17. 2007.

\section{Artigo de periódico}

AZUMA, R. T. Tracking Requirements for Augmented Reality, Communications of the ACM, p 50-51, July 1993

CARMO, R. M. C.; MEIGUINS, B. S.; GONÇALVES, A. S.; PINHEIRO, S. C. V.; ALMEIDA, L. H.; GODINHO, P. I. A. Coordinated and multiple views in augmented reality environment. In IV, p 156-162. IEEE Computer Society. 2007.

FERNANDES, B. C. A.; SANCHES, J. F. Realidade aumentada aplicada ao design. Holos, p 28 a 47. 2008.

GABBARD, J. L.; SWAN, E. Usability engineering for augmented reality: Employing user-based studies to inform design. IEEE Transactions On Visualization And Computer Graphics, p 513 - 525. 2008.

JONES, A.; SWAN, J. E.; SINGH, G.; KOLSTAD, E. The Effects of Virtual Reality, Augmented Reality, and Motion Parallax on Egocentric Depth Perception. Virtual Reality, p 267-268, IEEE, 2008.

ROGERS, P.; GRAHAM, C.; MAYES, C. Cultural competence and instructional design: Exploration research into the delivery of online instruction cross-culturally. Educational Technology Research and Development, p 197-217. 2007.

SCHOENFELDER, R.; SCHMALSTIEG, D. Augmented reality for industrial building acceptance. In Virtual Reality, p 83-90. IEEE. 2008.

ZORZAL, E. R.; OLIVEIRA, M. R. F.; SILVA, L. F.; CARDOSO, A.; KIRNER, C.; LAMOUNIER, E. Aplicação de Jogos Educacionais com Realidade Aumentada. CINTED-UFRGS. Novas Tecnologias na Educação. V.6 N¹ Julho 2008.

\section{Monografia, dissertação e tese}

AMADO, A. E. P. Avaliação de usabilidade de ambientes de realidade virtual e aumentada. Departamento de Electrônica Telecomunicações e Informática da Universidade de Aveiro, Portugal. 2007.

REBELO, I. B. Proposta de uma ferramenta de verificação dos procedimentos de interação em sistemas de realidade virtual. Universidade Federal de Santa Catarina Programa - Pós-Graduação em Engenharia e Produção, Florianópolis. 2004. 


\section{Trabalho em evento}

BACH, C.; SCAPIN, D. L. Obstacles and perspectives for evaluating mixed reality usability. V. 91 of CEUR Workshop Proceedings. CEUR-WS.org. 2004

BALCISOY, S.; KALLMANN, M.; FUA, P.; THALMANN, D. A. framework for rapid evaluation of prototypes with augmented reality. Proceedings of the ACM symposium on Virtual reality software and technology, p 61-66. 2000.

BASTOS, N. C. Uma metodologia para avaliação de usabilidade de interfaces de realidade mista interativas. XXXV Conferencia Latinoamericana de Informática. 2007

BONANNI, L.; LEE, C.H.; SELKER, T. Attention-based design of augmented reality interfaces, Portland, OR, USA. ACM, CHI '05 extended abstracts on Human factors in computing systems. 2005.

COSTABILE, M.; DE MARSICO, M.; LANZILOTTI, R.; PLANTAMURA, V.; ROSELLI, T. On the Usability Evaluation of E-Learning Applications, Proceedings of the 38th Annual Hawaii International Conference on System Sciences, 3-6 Jan. 2005.

DIAS, M.; JORGE, J.; CARVALHO, J.; SANTOS, P.; LUZIO, J. Usability evaluation of tangible user interfaces for augmented reality. Augmented Reality Toolkit Workshop, 2003. IEEE International. 2003.

JEON, S.; SHIM, H.; KIM, G. J. Viewpoint usability for desktop augmented reality. IJVR, p 33-39. 2006

KULAS, C. S. C.; KLINKER, G., MÄUNCHEN, T. U.; SOFTWARETECHNIK, L. F. A. Towards a development methodology for augmented reality user interfaces. The International Workshop exploring the Design and Engineering of Mixed Reality Systems - MIXER 2004.

LUZ, R.; RIBEIRO, M. W. S.; CARDOSO, A.; LAMOUNIER Jr., E.; ROCHA, H.; SILVA, W. Análise De Aplicações De Realidade Aumentada Na Educação Profissional: Um Estudo De Caso No Senai Dr/Go. 5o. Workshop de Realidade Virtual e Aumentada - WRVA'2008

POLYS, N. F.; BOWMAN, D. A. Design and display of enhancing information in desktop information-rich virtual environments: challenges and techniques. Virtual Reality, p 41-54. 2004.

SOUZA, L.; SPINOLA, M. Requisitos de usabilidade em projetos de interface centrado no usuário de software de dispositivos móveis. XXVI ENEGEP. 2006.

SWAN, J. E.; GABBARD, J. L. Survey of user-based experimentation in augmented reality. 1st International Conference on Virtual Reality, HCI International 2005, Las Vegas, Nevada, USA. 2005.

VANDERDONCKT, J.; CHIEU, C. K.; BOUILLON, L.; TREVISAN, D. Model-based design, generation, and evaluation of virtual user interfaces. The ninth international 
conference on 3D Web technology (WEB3D'04), p 51-60, New York. ACM Press. 2004.

\section{Internet}

ISO. Norma Padrão Internacional ISO 13407. 1999. International Standards for Business, Government and Society. Disponível em: <www.iso.org/cate/d21197.html> Acesso em: 15 junho 2008. 